\title{
Study of blood flow impact on growth of thrombi using a multiscale model
}

\author{
Zhiliang Xu, ${ }^{* a}$ Nan Chen, ${ }^{a}$ Shawn C. Shadden, ${ }^{b}$ Jerrold E. Marsden, ${ }^{c}$ Malgorzata M. Kamocka, ${ }^{d}$ \\ Elliot D. Rosen ${ }^{d}$ and Mark Alber ${ }^{* a}$
}

\author{
Received 21st July 2008, Accepted 3rd October 2008 \\ First published as an Advance Article on the web 12th December 2008 \\ DOI: $10.1039 / \mathrm{b812429a}$
}

\begin{abstract}
An extended multiscale model is introduced for studying the formation of platelet thrombi in blood vessels. The model describes the interplay between viscous, incompressible blood plasma, activated and non-activated platelets, as well as other blood cells, activating chemicals, fibrinogen and vessel walls. The macroscale dynamics of the blood flow is represented by the continuous submodel in the form of the Navier-Stokes equations. The microscale cell-cell interactions are described by the stochastic Cellular Potts Model (CPM). Simulations indicate that increase in flow rates leads to greater structural heterogeneity of the clot. As heterogeneous structural domains within the clot affect thrombus stability, understanding the factors influencing thrombus structure is of significant biomedical importance.
\end{abstract}

\section{Introduction}

The hemostatic system has evolved to prevent the loss of blood at the site of vascular injury. The response is rapid to limit bleeding and is regulated to prevent excessive clotting that can limit flow. The processes that are involved in the assembly of a thrombus (blood clot) include complex interactions among multiple molecular and cellular components in the blood and vessel wall occurring under fluid flow.

Formation of a thrombus (thrombogenesis) involves the close interplay between many processes that occur at different scales (subcellular, cellular and multicellular, et al.). In the past, these processes have been studied separately.

The flowing bloodstream over the thrombus affects its growth and development. Blood carries cellular and molecular components to the thrombus for their incorporation into the structure. These not only include coagulant and anticoagulant proteins but platelets and blood cells. While histologic analysis of thrombi formed in vivo reveals the incorporation of leukocytes and erythrocytes, most models of thrombogenesis fail to include these cells, viewing them as passive elements entrapped in the clot. On the other hand, recent evidence suggests that cellular derived micro-particles in blood are also required for thrombus formation.

Furthermore, shear forces generated by the flowing blood can dislodge elements from the structure. Moreover, the growing thrombus may generate turbulence in the flow field with significant consequences to growth properties. Vortices forming behind the thrombus can trap platelets and blood cells and move them back towards the clot. This entraps blood cells producing a thrombus with heterogeneous domains that may affect clot

${ }^{a}$ Department of Mathematics, University of Notre Dame, Notre Dame, IN, 46556,USA.E-mail:malber@nd.edu; zxu2@nd.edu

${ }^{b}$ Cardiovascular Biomechanics Research Laboratory, Stanford University, Palo Alto, CA, 94305, USA

${ }^{\circ}$ Department of Control and Dynamical Systems, CaltechPasadena, CA, 91125, USA

${ }^{d}$ Department of Medical and Molecular Genetics, Indiana University School of Medicine, Indianapolis, IN, 46202, USA stability. Hemodynamic parameters also affect processes at the cellular and subcellular scale. Endothelial cells respond to changes in flow. Not only does flow affect cell shape, but it also influences gene expression patterns. The pattern of gene expression in endothelial cells cultured under flowing media in vitro is more typical of resting cells, while inflammatory markers are expressed by cells growing under static conditions.

Whereas most existing models describe continuous dynamics at the macroscale related to platelet aggregation and coagulation reactions, there is a reason to believe that many aspects of the cascade are better modeled as discrete events at the microscale. First, certain enzymes exhibit threshold effects on the activation or inhibition of substrate proteins. Second, some of the more complex aspects of the coagulation process are poorly understood, and certainly there is no closed-form set of differential equations for the system as a whole. Moreover, more coarse-grained information is available at the microscale, e.g., whether a certain protein factor must be present at some minimal concentration for a reaction to take place, and it can be incorporated into the model in the form of discrete states.

Cell-based discrete models are used in a variety of problems dealing with biological complexity. One motivation for this approach is the enormous range of length scales of typical biological phenomena. The recent book ${ }^{3}$ reviews many of the cellbased models. Treating cells as simplified interacting agents, one can simulate the interactions of tens of thousands to millions of cells and still have within reach the smaller-scale structures of tissues and organs that would be ignored in continuum (e.g., partial differential equation) approaches. At the same time, discrete stochastic models including the Cellular Potts Model (CPM) can be made sophisticated enough to reproduce almost all commonly observed types of cell behavior. ${ }^{11-13,19,36-37}$ The CPM, which is an extension of the Potts model from statistical physics, can be made sophisticated enough to reproduce almost all commonly observed types of cell behavior. It has become a common technique for simulating complex biological problems including embryonic vertebrate limb development, ${ }^{12,23}$ tumor growth ${ }^{17}$ and vasculogenesis. ${ }^{22}$ 
We believe that various aspects of the development of thrombi are best studied using a multiscale hybrid model combining discrete and continuous submodels at different scales. In ref. 38 the authors introduced a basic multiscale two-dimensional model of thrombus formation. The model combines submodels of all interrelated factors that contribute to the formation of a blood thrombus at three spatial scales: 1) the vessel wall scale, assuming the diameter of the artery to be of the order of 100 microns, 2) the cellular scale determined by the diameter of a platelet being of the order of 1 micron, and 3 ) the inter-cellular scale of the fibrin fibril network.

The stochastic CPM is used in this model to represent several types of cells of different sizes and with different adhesivity properties as well as to describe platelet-injury adhesion, platelet activation, platelet-platelet binding, cell movement, cell state changes and platelet aggregation. The incompressible NavierStokes (NS) equations describe dynamics of viscous blood plasma and the interface submodel couples the CPM with the continuous model to describe the thrombus-blood plasma interface. The CPM and the blood flow model respectively are defined on the lattice and the volume that fills the finite difference grid. The two computational grids are spatially superimposed.

In this paper, we further develop this multiscale model by introducing additional types of blood cells and use it to study clot formation under various flow conditions. In particular, we describe the impact of flow shear stresses on the final positions in the thrombi of the cells with different adhesive properties. Furthermore, we employ the Lagrangian Coherent Structure (LCS) method (these methods have a long history, with some related techniques going back to ref. 26. We follow the method of Haller, ${ }^{16}$ see the history of this subject in ref. 33 and in ref. 20) to analyze the detailed flow structure near the clot to reveal the role of blood flow in clot formation.

The paper is organized as follows. Section 2 provides biological background on thrombus development. Section 3 presents a brief overview of the extension of the multiscale computational model. Section 4 contains an extended discussion of simulations and model parameter selection, demonstrates the robustness of the model and describes blood flow effects on the growth of thrombus. Section 5 contains the conclusions.

\section{Biological and modeling background}

Following vascular injury, blood is exposed to prothrombotic environments that promote rapid thrombus formation. Thrombus formation is the result of two interrelated processes, namely, platelet interactions and activation of the coagulation pathway. Immediately after vessel damage, platelets adhere to the site of vessel injury forming a single cell layer. Platelet adhesion is promoted by the binding of platelet receptors GpIb/ V/IX to von Willebrand factor (vWF) and GpIa/IIa and GpVI to collagen in the subendothelial matrix of the vessel wall. However, more limited endothelial damage which doesn't denude the endothelium and expose the subendothelial matrix also promotes platelet binding. Conceivably, activated endothelial cells expose long vWF multimeric chains released from endothelial WeibelPalade bodies.

Following adhesion, platelets form multicellular aggregates mediated by the binding of the platelet GpIIb/IIIa receptor to fibrin(ogen). In addition, platelets undergo activation involving significant morphological changes, the exposure of new proteins on the platelet surface and the extracellular release of contents of alpha and dense granules found in resting platelets. These contents include a variety of hemostatic proteins and molecules that stimulate a variety of cells at the site of vessel injury. Activated platelets also provide a phospholipid surface that promotes the surface dependent coagulation reactions at the site of injury.

In addition to platelet interactions, coagulation factor VII(a) in the blood is exposed to its cellular receptor, TF, expressed on cells in the vessel wall. Coagulation is initiated when FVIIa binds to TF. The TF/FVIIa complex catalyzes conversion of FX to FXa and FIX to FIXa, and initiates a complex network of reactions that lead to rapid generation of thrombin, which serves to convert fibrinogen to fibrin, the major matrix protein in a thrombus, and activates FXIII to cross-link fibrin. In addition, thrombin triggers a positive feedback loop of propagation reactions. Thrombin is also a potent activator of resting platelets and thus mediates recruitment of resting platelets owing nearby in the blood. The activated platelets provide a procoagulant surface that promotes coagulation enzyme activity. Making matters more complex, thrombin also activates the Protein $\mathrm{C}$ anticoagulant pathway that inhibits the loop of propagation reactions by inactivating coagulation factors $\mathrm{FVa}$ and FVIIIa.

In the past, subprocesses involved in thrombogenesis have been studied as distinct disciplines. For example, the extrinsic and intrinsic coagulation pathways have been modeled using the feedback loops described in ref. 18 and 4. The multifaceted models have been developed based on a large number of reaction-diffusion equations with parameters related to many aspects of clot growth such as flow rates, membrane binding site density and concentration of calcium (see ref. 40,39,25). Several models concentrated on specific aspects of the coagulation. In ref. 6 and 7 it was shown that activation threshold for an enzyme cascade was affected by the flow rate, the size of the injury and initial concentrations of active enzymes. The regulation of activation threshold by levels of Factor-VII and XII and clotting time sensitivity to zymogen concentrations has been studied using spatially homogeneous and inhomogeneous systems. Ref. 5 and 40 studied clot growth and dissolution using spatially inhomogeneous systems.

There are a few constitutive models of clot formation as well. A 1D linear viscoelastic clot model has been proposed, ${ }^{21}$ a 3-D Maxwell model was developed for the study of coagulation of whole blood, ${ }^{28}$ and a Newtonian fluid model in the form of coupled convection-reaction-diffusion equations without platelet deposits has been used. ${ }^{14,35}$ These models neglected the effect of the growing clot on the flow field. A recent model ${ }^{2}$ incorporated both rheological properties of the clot and multiple biochemical reactions. They modeled clot formation and dissolution as the growth/diminishment of a singular front (viscoelastic liquid clot) in a sheer-thinning viscoelastic whole blood region. The convection-reaction-diffusion equations have been used for modeling platelet activation, extrinsic coagulation pathway and fibrinolysis. Due to the extremely complicated nature of blood clotting, these attempts usually focused on small subsets of the entire process. 


\section{Multiscale computational model}

Due to the multiscale nature of platelet aggregation and coagulation reactions, capturing both the discrete events and the continuous mechanics, of thrombus development is best modeled as a hybrid composition of the two. The multiscale approach takes advantage of relative simplicity and efficiency of the macroscopic models, as well as the accuracy of the microscopic models, and it is particularly helpful for studying the macromicro relationships of blood flow and platelet aggregation and coagulation. It allows one to model in detail formation of the thrombus as well as a boundary layer between thrombus and blood flow, resulting in a description of different phases in thrombus development and of mechanisms determining its shape and internal structure.

\subsection{Submodels at different scales}

Our model consists of a set of submodels for processes involving components at scales described in the previous section:

- Biochemical reaction submodel: we use a set of PDEs to describe the net effect of biochemical reactions.

- Cell submodel: The discrete stochastic CPM represents different types of cells as well as accounts for platelet-injury adhesion, platelet activation, platelet-platelet binding, cell movements, cell state changes and platelet aggregation.

- Flow submodel: The incompressible Navier-Stokes equations describe dynamics of viscous blood plasma.

- Interface submodel: The submodel couples the CPM with the continuous model to describe the thrombus-blood plasma interface. The CPM and the blood flow model are defined on the lattice and volume filling finite difference grid respectively. Two computational grids are spatially superimposed.

The simulation of the thrombus development starts with utilizing macroscale submodules involving optimized NavierStokes and advection diffusion solvers. Subsequently, obtained blood flow velocity field and thrombin distribution is fed into the CPM describing individual cell behavior. On the other hand, cell differentiation and cell state transition is determined based on the chemical concentration derived from a sub-cellular module based on the kinetic equations modeling coagulation pathway. This information is used when simulating generation of fibril which eventually form fibrin network. We assume that geometry of the thrombus (boundary conditions) does not change considerably during calculation of the flow velocity field. The NS equations and advection-diffusion equations for the flow and chemical distributions are solved with this boundary condition provided by the CPM. The result in turn is used in the subsequent CPM update. It is also assumed that during the CPM step flow conditions (velocity and pressure) remain unchanged. The updated CPM data (interface geometry and chemical concentrations) provide new boundary and initial conditions for the NS advection-diffusion equations modules. The spatial coupling between cellular and thrombus scales is achieved through mesh refinement between the CPM cell lattice and the finite difference grid. The chemical concentration, obtained from the sub-cellular kinetic equations, is averaged to these grids to reduce the computational complexity.

\subsection{Stochastic cellular Potts model}

The CPM is a cell-level, energy-minimization-based lattice model, which uses an effective energy $E$ coupled to external fields, e.g., the local flow rate, to describe cell motion, cell-cell interactions, cell adhesion, differentiation, division and hapoptosis. Each cell consists of many pixels (lattice sites). The distribution of multidimensional indices associated with lattice sites determines current system configuration. The effective energy of the system mixes true energies, like cell-cell adhesion, and terms that mimic energies, e.g., the response of a cell to a blood flow and the constraint

$$
E=E_{\text {adhesion }}+E_{\text {flow }}+E_{\text {dim }}
$$

Given an effective energy one can calculate the resulting cell motion using Metropolis dynamics algorithm based on the Monte-Carlo Boltzmann acceptance rule. ${ }^{24}$ More details of the energy form used in the clot formation modeling can be found from our previous publication. ${ }^{38}$

Our model involves eight types of cells (see Fig. 1). "Injury" and "Vessel" cells do not represent cells in the CPM and are used only to indicate boundary of the vessel domain and place of injury. During simulation, their positions and adhesion properties are fixed and platelets can adhere to their surfaces. New platelets (inactivated) and blood cells are injected from the inlet (left) side at a specified rate. When cells reach the outlet (right) side of the simulation domain, they are removed from the system. We impose the "no-flux" boundary condition on both upper and lower boundaries so that cells would not move outside of the domain.

When an inactivated platelet reaches the cite of the injury or the ADP concentration of a platelet becomes higher than the set threshold value, it changes its type to "activated" and starts releasing thrombin which causes fibril generation. Generated fibrils gradually form fibril network which increases thrombus stiffness and prevents platelets from detaching from the thrombus surface. The fibril level in the thrombus and activated platelet cluster increases with the increase in thrombin concentration. Thus, clusters consisting of activated platelets, have different fibril levels and stiffness properties at initial and later stages. In our CPM, we implement two different activated platelet states (activated platelet and activated platelet with high fibril level), with different flow responses. ${ }^{38}$

Platelet adhesion is mediated by plasma proteins bridging receptors on adjacent platelets (gpIIbIIIa fibrinogen, vironecting, von Willebrand Factor, gpV1-collagen, etc). It is difficult to describe all details of cell-cell adhesion in the model. In the CPM the cell-cell adhesion is described as an energy penalty function term: low cell-cell adhesion energy represents strong adhesivity between cells while high cell-cell adhesion energy represents weak cell-cell adhesivity.

In the CPM, the adhesion energy is calculated by

$$
E_{\text {adhesion }}=\sum_{(i, j, k),\left(i^{\prime}, j^{\prime}, k^{\prime}\right) \text { neighbors }} J_{\tau(\sigma) \tau^{\prime}\left(\sigma^{\prime}\right)}\left(1-\delta\left(\sigma(i, j, k), \sigma^{\prime}\left(i^{\prime}, j^{\prime}, k^{\prime}\right)\right)\right),
$$

where $\delta\left(\sigma, \sigma^{\prime}\right)=0$ if $\sigma \neq \sigma^{\prime}$, and $\delta\left(\sigma, \sigma^{\prime}\right)=1$ if $\sigma=\sigma^{\prime}$. These conditions ensure that only the surface sites between different 
cells contribute to the adhesion energy. Each term under summation sign is a product of the binding energy per unit length, $J_{\tau(\sigma) \tau^{\prime}\left(\sigma^{\prime}\right)}$, and the length of a contact between two neighboring cells. Adhesive interactions act up to second-nearest-neighbors. Ref. 15, 27 and 29 show that the adhesivity between platelets and thrombus is strongly affected by the flow shear rate on the thrombus surface. At low shear rate, the value of adhesivity is low and it becomes higher with the increase of the shear rate, eventually to a constant value. To describe the dependence of the adhesivity on the shear rate, $J_{\tau(\sigma) \tau^{\prime}\left(\sigma^{\prime}\right)}$ is described as a function of the flow shear rate $\dot{\gamma}$ on the thrombus surface:

$$
J_{\tau(\sigma) \tau^{\prime}\left(\sigma^{\prime}\right)}(\dot{\gamma})=\left\{\begin{array}{cc}
J_{h}+J_{e} \frac{\dot{\gamma}_{h}-\dot{\gamma}}{\dot{\gamma}_{h}-\dot{\gamma}_{l},} & \dot{\gamma} \leq \dot{\gamma}_{t 1} \\
J_{h} & \dot{\gamma} \leq \dot{\gamma}_{t 1}
\end{array}\right.
$$

where $\dot{\gamma}_{h}$ and $\dot{\gamma}_{l}$ are the high and low thresholds values of the shear rate, and $J_{h}$ and $J_{e}$ are adhesion energy constants.

\subsection{Continuous submodels of blood flow and biochemical reactions}

Blood is a complex mixture of cells, proteins, lipoproteins and ions by which nutrients and wastes are transported. Red blood cells comprise approximately $40 \%$ of blood by volume. In contrast to water, blood does not exhibit a constant viscosity at all flow rates and is non-Newtonian in the microcirculatory system (in small branches and capillaries). The normal blood flow is laminar with secondary flows generated at curves or branches. The Reynolds number varies from 1 in small arterioles to approximately 4000 in the largest artery. Moreover, the blood flow and pressure are unsteady, since the heart creates pulsating conditions in all arteries. Depending on the artery and the flow being modeled, the following main features are usually addressed in a comprehensive blood modeling effort: vessel wall elasticity, non-Newtonian viscosity, Fahraeus-Lindquist effect, motion of particles in the fluid, Fahraeus effect, body forces, and temperature. The Fahraeus-Lindquist effect accounts for the blood viscosity dependence on the diameter of the vessel it travels through. The Fahraeus effect is present when blood flows from a large reservoir into a small circular cylindrical tube. As a result the hematocrit in the tube is smaller than that in the reservoir.

Since we model the development of thrombus inside a blood vessel of length approximately 10 times larger than the its diameter, in our model, we simplify the vessel to be a straight pipe. The vessel wall is assumed to be a rigid wall. Blood plasma is treated as an incompressible fluid, which is modeled by the two-dimensional incompressible Navier-Stokes equations that take the form

$$
\begin{gathered}
\frac{\partial \vec{u}}{\partial t}+(\vec{u} \cdot \nabla) \vec{u}+\frac{1}{\rho} \nabla p=\frac{\mu}{\rho} \nabla^{2} \vec{u}+\vec{f} \\
\nabla \cdot \vec{u}=0
\end{gathered}
$$

where $\vec{u}=(u, v)$ is the flow velocity, $\rho$ is the density of the blood plasma, $p$ is the pressure, and $\mu$ is the viscosity. We impose the Dirichlet boundary condition $(u, v)=(0,0)$ on no-slip boundaries.

The major goal of the paper is to study how blood flow affects developing clots. For this reason, we apply a simple phenomenological model to describe the biochemical processes in the form of the following system of reaction advection-diffusion equations ${ }^{35}$

$$
\frac{\partial C_{k}}{\partial t}+\nabla \cdot\left(C_{k} \vec{u}\right)=D_{k} \nabla^{2} C_{k}+S_{k}, \quad k=1,2, \ldots, 5
$$

where $D_{k}$ is a diffusion coefficient of the $k$-th chemical component. $S_{k}$ is the reaction source term of the $k$-th chemical component due to the enzymatic reactions. This model is comprised of equations describing platelet released agonist, adenosine diphosphate (ADP), and synthesized agonist, thromboxane (TxA2), which can be inhibited via 1st order reactions; prothrombin; thrombin; and antithrombin III (ATIII), which inhibits thrombin.

Table 1 lists coefficients of chemical components used in the equations, the source terms and initial concentrations in blood. Here symbol $C_{k}$ represents chemical concentration, [AP] and [RP] are concentrations of the activated and resting platelets, which are computed from the discrete CPM. (See ref. 35 for the parameter values appearing in the source term.)

The activation condition for the platelets depends on the concentrations of the chemical components as well as blood flow conditions. Platelet activation is partially triggered by the exposure to shear stresses which are higher than certain threshold values. ${ }^{10}$ In the problems studied in the paper, stresses are lower than the threshold values.

We assume that activation of platelets occurs when the concentration of chemical components is higher than a specified threshold. The valid activator concentration is calculated according to: ${ }^{35}$

$$
\Omega=\frac{[\mathrm{ADP}]}{\left[\mathrm{ADP}_{\text {crit }}\right]}+\frac{[\mathrm{TxA} 2]}{\left[\mathrm{TxA}_{\text {crit }}\right]}+\frac{[\text { Thrombin }]}{\left[\text { Thrombin }_{\text {crit }}\right]}
$$

where $a_{\text {crit }}$ is the threshold concentration of that agonist for platelet activation, which is obtained from ref. 35 . Once the valid activator concentration $\Omega$ around a platelet is higher than the threshold value $T_{a}$, the platelet is activated.

Table 1 Coefficients of chemical components and source terms of equations

\begin{tabular}{llll}
\hline Species & $D_{i} / \mathrm{cm}^{2} \mathrm{~s}^{-1}$ & $S_{i}$ & Init. Conc. \\
\hline ADP & $2.56 \times 10^{-6}$ & $\lambda_{\mathrm{i}} k_{\mathrm{pa}}[\mathrm{RP}]-k_{1, j}[\mathrm{ADP}]$ & $0.0 \mu \mathrm{M}$ \\
TxA2 & $2.14 \times 10^{-6}$ & $S_{\mathrm{pj}}[\mathrm{AP}]-k_{1, j}[\mathrm{TxA} 2]$ & $0.0 \mu \mathrm{M}$ \\
Prothrombin $[\mathrm{PT}]$ & $3.32 \times 10^{-7}$ & $-\beta[\mathrm{PT}]\left(\phi_{\mathrm{at}}[\mathrm{AP}]+\phi_{\mathrm{rrt}}[\mathrm{RP}]\right)$ & $1.0 \mu \mathrm{M}$ \\
Thrombin [T] & $4.16 \times 10^{-7}$ & $-\Gamma[T]+[\mathrm{PT}]\left(\phi_{\mathrm{at}}[\mathrm{AP}]+\phi_{\mathrm{rt}}[\mathrm{RP}]\right)$ & $0.0 \mathrm{U}$ \\
ATIII & $3.49 \times 10^{-7}$ & $-\Gamma \beta[T]$ & $2.844 \mu \mathrm{M}$ \\
\hline
\end{tabular}


3.3.1 Parabolic and pulsatile flow models. There is evidence that vascular fluid dynamics plays an important role in the development of the thrombus. To mimic physiological flow conditions, we used a sinusoidal time-dependant velocity function to model the effect of pulsatility. We impose on the inlet section that

$$
u=u_{0}(1.0+\varepsilon \sin (w t))
$$

where $u_{0}$ is mean velocity and $\varepsilon$ is an amplitude of the pulsatility relative to the mean velocity. The period of oscillation is $w=1 \mathrm{~s}$. We also assume that the upstream flow is laminar and has a parabolic profile, resulting in the following inlet velocity

$$
\begin{gathered}
u(h)=u\left[1.0+1.44 \sqrt{f}+2.15 \sqrt{f} \log _{10}\left(1-\frac{2 h}{D}\right)\right], \\
v=0.0
\end{gathered}
$$

where $u(y)$ is a $x$-velocity component at a distance $h$ from the center of the pipe on the inlet, $v$ is a $y$-velocity component, $D$ is diameter of the pipe and $f$ is the friction factor, which is set to be $f$ $=0.01$. In general, the friction factor depends on the Reynolds Number of the pipe flow. Here we assume that the Reynolds Number is moderate and the inner side of the blood vessel is smooth in the upstream flow direction.

3.3.2 Non-Newtonian blood viscosity model. To identify the role of the non-Newtonian property of the blood, we implemented widely used Carreau-Yasuda model $^{8}$ for the blood viscosity

$$
\mu(\dot{\gamma})=\mu_{\infty}+\left(\mu_{0}-\mu_{\infty}\right)\left[1+(\lambda \dot{\gamma})^{a}\right]^{(n-1) / a}
$$

where viscosity $\mu$ is a function of the shear rate $\dot{\gamma}=\sqrt{2 \underline{\underline{D}}: \underline{\underline{D}}}$ with the following rate of deformation tensor

$$
\underline{\underline{D}}=\frac{1}{2}\left(\nabla \vec{u}+(\nabla \vec{u})^{T}\right) .
$$

Here $a, n$ and $\lambda$ are empirically determined parameters.

In this paper we use the following values $a=2, n=0.3568, \lambda=$ 3.313 s. Parameters $\mu_{\infty}$ and $\mu_{0}$ are chosen as $\mu_{\infty}=0.0345 \mathrm{~g} \mathrm{~cm}^{-1}$ $\mathrm{s}^{-1}$ and $\mu_{0}=0.56 \mathrm{~g} \mathrm{~cm}^{-1} \mathrm{~s}^{-1}$ respectively. These parameter values were taken from a study of the pulsatile flow of blood through stenotic arteries. ${ }^{1}$ The calibration of these parameter values was carried out by a simulation study to show the rheological behavior of blood flow and to calculate the magnitude of the wall shear stresses. In simulations with blood being treated as Newtonian flow, the viscosity $\mu$ value is set at $\mu=0.04 \mathrm{~g} \mathrm{~cm}^{-1} \mathrm{~s}^{-1}$ based on the experimental data.

\subsection{Coupling between continuous and discrete submodels}

3.4.1 Thrombus-plasma interface. We use volume of fluid approach to explicitly track the interface between developing the thrombus (or platelet aggregates at an earlier stage) and blood plasma. A thrombus, in principle, can be treated as a gel-like matter, and exhibit viscoelastic behavior. In this paper, we make the following assumptions to simplify the description of the interactions:
- Blood plasma (the liquid phase) and the thrombus (viscoelastic matter phase) are assumed to be separated by a sharp interface called thrombus-plasma interface.

- It is also assumed that within a time step of the NavierStokes PDE solver, the thrombus domain does not change.

Based on these assumptions, we solve Navier-Stokes equations to update the flow velocity and pressure fields inside thrombus domain which is fixed during a time step of the PDE cycle. The updated velocity and pressure fields, in turn, drive the cells' motion and aggregation, resulting in thrombus shape change during the subsequent CPM time step.

All cells in the simulation domain are divided into two types: cells floating in blood stream and cells on the thrombus. We use two different methods to handle cell-fluid interactions for two types of cells. Floating cells are not connected to each other and it would be very computationally expensive to take all individual cell geometries into account in solving the Navier-Stokes equation. The effects of the small floating cell on the blood velocity distribution are very weak and are ignored in our model to save calculation time. We do not take floating cells into account in the boundary condition setup for Navier-Stokes equations. The motion of floating cells is calculated from the blood velocity distribution and the flow energy of floating cells depends on the blood velocity. The cells that are located on the thrombus are connected to each other by fibers and are treated as a stiff obstacle in solving the Navier-Stokes equations. Since they are treated as the stiff boundary, the blood velocities for thrombus cells are zero and the motion of them cannot be determined from the blood velocity. We use the blood pressure to determine the thrombus cells' motion, and the flow energy term of the thrombus cells depends on blood pressure. The blood pressure on the cells that are located on the thrombus boundary is nonzero and drives them to move towards the interior of the thrombus, compressing internal thrombus cells. However, the volume constraint on the internal thrombus cells resists the compression from the outside cells and the balance between two trends is obtained from the energy-minimizing principle of the CPM.

The evolution of the interface between thrombus and blood plasma is driven by the platelet aggregation and coagulation and its dynamics is described by the CPM. To track the interface, we superimpose the CPM cell lattice (grid) on and align with the PDE grid (see Fig. 2).

The thrombus-plasma interface is identified on the CPM lattice as a union of outer boundaries of cells located in the current surface of a thrombus. The interface is projected onto the PDE grid to provide boundary conditions for the PDE solution step. We employ no slip boundary conditions.

3.4.2 Impact of the blood flow on a moving cell. We use the flow energy term in the CPM Hamiltonian to describe the impact of the blood flow on the cells. The flow energy terms for different types of cells are calculated from the flow velocity distribution or flow pressure.

Cells with high level of fibril concentration are very stiff and aggregate together to form the thrombus. In this model, they are treated as obstacles for the blood flow. The flow force applied to one of these type cells is calculated as an integral of blood pressure along a cell membrane: 


$$
\vec{F}_{i}=\sum_{\substack{\text { All cell-blood interface } \\ \text { segments } k \text { of cell } i}} p_{k} \cdot \vec{n}_{k} S_{k}
$$

where $p_{k}$ is the pressure applied to the blood-cell interface segment $k, \vec{n}_{k}$ is the inward unit normal of blood-cell interface segment $k$, and $S_{k}$ is the membrane length of the blood-cell interface segment $k$. For a state change attempt, the flow energy change for the cell $i$ is

$$
\Delta E_{\text {flow }(i)}=-K_{e 1} \vec{F}_{i} \cdot \Delta \vec{d}_{i}
$$

where $\Delta \vec{d}_{i}$ is the change in the position of the center of mass of cell $i$ caused by the state change attempt and $K_{e 1}$ is a flow energy constant.

The highly mobile cells (e.g. resting platelets) are separately distributed in the blood flow and it would be computationally expensive to calculate the flow distribution around each individual cells using the Navier-Stokes equation. The size of these types of cells is very small and the effects of them on the flow are negligible. We assumed that these cells have the same velocity as the those in the flow. Thus, the average flow velocity for a cell $i$ is

$$
\vec{V}_{i}=\sum_{\text {All pixels of cell } i} \vec{v}_{k} / \mathrm{Vol}_{i}
$$

where $\vec{V}_{i}$ is the velocity of cell $i, \vec{v}_{k}$ is the flow velocity at cite $k$ and $\mathrm{Vol}_{i}$ is the volume of cell $i$. The flow energy change for cell $i$ caused by state change is equal to

$$
\Delta E_{\text {flow }(i)}=-K_{e 2} \vec{F}_{i} \cdot \Delta \vec{d}_{i}
$$

where $\Delta \vec{d}_{i}$ is the change of the center of mass of cell $i$ caused by the state change and $K_{e 2}$ is a flow energy constant.

3.4.3 Coupling time scales of continuous models and CPM. The CPM is an extension of the Potts model which does not explicitly indicate its physical time scale. The time scale is imposed on the CPM by matching the simulated dynamics with the real physical one. In this model, the moving velocity of a platelet is assumed equal to the blood value calculated from the Navier-Stokes equation. Thus the time scale between the CPM and the continuous model is established by matching the simulated platelet moving velocity with the blood flow velocity. During the parameter calibration process, we gradually adjust the flow energy constant value in the CPM until the platelet moving velocity matches the blood's. Using the calibrated flow energy constant ensures that the CPM and the continuous model are in the same time scale.

\subsection{Analysis of the blood flow}

To study transport during the formation of the clot we make use of Lagrangian coherent structures (LCS). For the present analysis, constituents in the clotting process are assumed to be transported by the flow generated by the velocity field of the fluid. That is, letting $\vec{v}(\vec{x}(t), t)$ denote the velocity field obtained from solving the Navier-Stokes equations, the key constituents in the blood are assumed to be transported according to

$$
\vec{x}(t)=\vec{v}(\vec{x}(t), t)
$$

This assumption is reasonable based on the timescale of the dynamics considered, and based on the size and mass of the cells and proteins affecting clot formation.

Letting $\phi(\vec{x}(t), t, \tau): \vec{x}(t) \rightarrow \vec{x}(t+\tau)$ denote the flow map generated by integrating eqn (3.17), we can define the finite-time Lyapunov exponent (FTLE) as

$$
\sigma(\vec{x}, t, \tau)=\frac{1}{\tau}\left\|\frac{\mathrm{d} \phi(\vec{x}, t, \tau)}{\mathrm{d} \vec{x}}\right\|
$$

Here the norm $\|A\|$ of a matrix $A$ is defined as $\|A\|^{2}=\lambda_{\max }\left(A^{*} A\right)$, where $\lambda_{\max }$ is the max eigenvalue. The spatial distribution of the FTLE can be used to define separatrices in the flow known as LCS. ${ }^{26,16,33,20}$ Under this framework LCS are defined as curves that locally maximize the FTLE in the direction normal to the curve. ${ }^{33}$ A positive integration time (i.e. $\tau>0$ ) used to compute the FTLE field reveals a repelling LCS, whereas integrating the particle position backward in time $($ i.e. $\tau<0)$ reveals an attracting LCS in the FTLE field. Often, LCS defined in this manner are not sensitive to variations in integration time. However, when the flow becomes turbulent and the flow structure changes rapidly, more care must be taken in choosing an appropriate integration time. More details about the choice and influence of the integration time can be found in ref. 33,30 and 20.

The advantage of this method is that LCS defines boundaries between regions of fluid that have distinct dynamics. This can help to identify the boundary of recirculation regions, vortex boundaries, or where separation or reattachment of the flow occurs in a precise manner. Such concepts are difficult or ambiguous to define using standard criteria in flow analysis when the system is unsteady, see e.g. ref. $31,32,34$.

\section{Simulation results}

In this section we describe simulations which have been performed to study clot development under various flow conditions. Table 2 lists simulation parameter values.

The coagulation reaction pathway reaction rates and initial chemical concentrations are from ref. 35 and 9 . We used the $\Omega$ (activation function) from ref. 35 to determine the platelet state transition in Fig. 3.

\subsection{Clot growth under parabolic flows}

To validate the computational model, we compared the simulation results with the experiments conducted by Begent and Born, ${ }^{9}$

Table 2 Simulation parameters

Quiescent platelet-quiescent

20.0
8.0
15

2.0
$K_{\mathrm{e} 1}=25.0, K_{\mathrm{e} 2}=0.2$
1.0
6.0
$250 \mu \mathrm{m} \times 25 \mu \mathrm{m}$
$1250 \times 125$

Activated platelet-activated platelet adhesion energy

Activated platelet (high fiber level-low fiber level) adhesion energy

Volume energy constant

Fluid energy constant

Threshold1

Threshold2

Simulation domain

CPM grid size
$1250 \times 125$ 


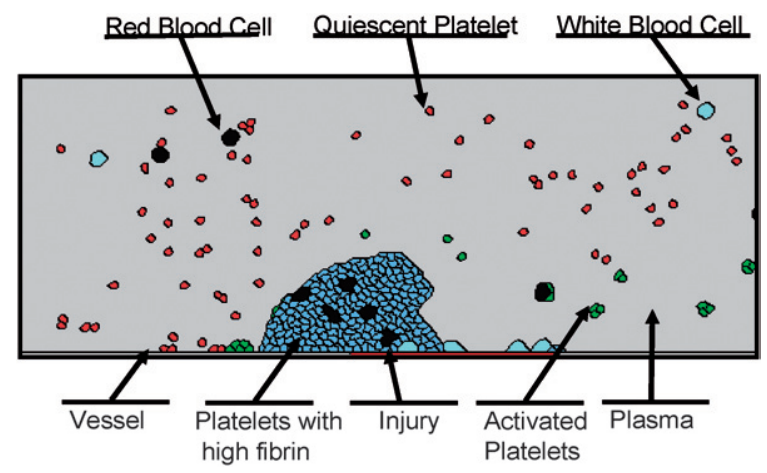

Fig. 1 Different types of cells in the model of thrombus development.

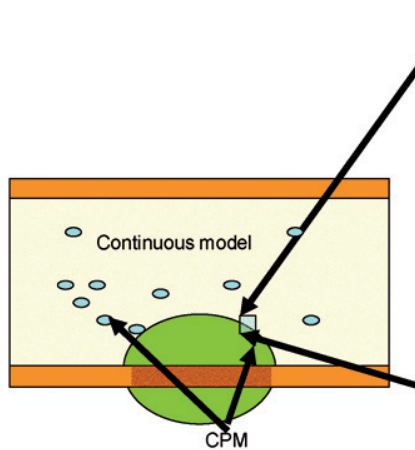

(a)

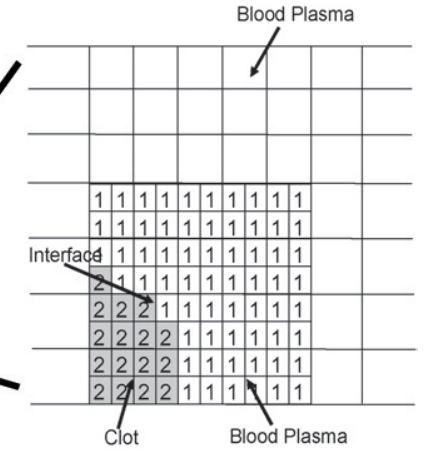

(b)
Fig. 2 Diagram of the thrombus-plasma interface. a) The thrombusplasma interface in the simulation. b) Spatially coupled finite difference grid and the CPM lattice.

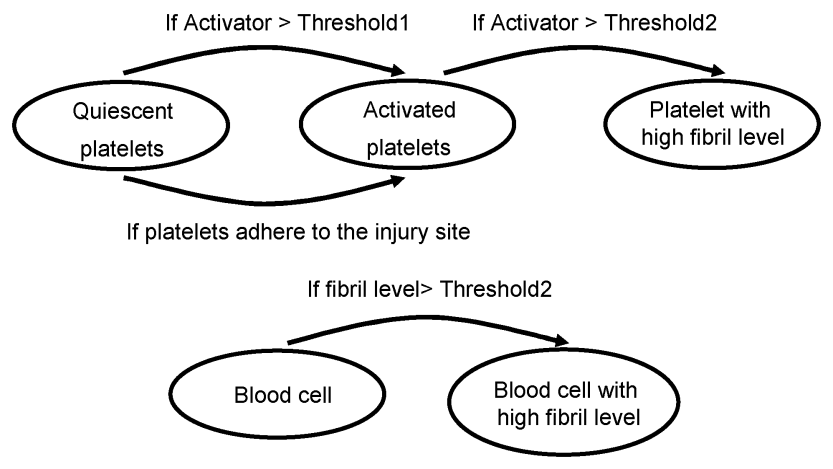

Fig. 3 Cell state transition map.

in which they found that the size of the resulting thrombus first increased and then decreased with increase of blood flow velocity. We ran simulations with different mean blood flow velocities ranging from $200 \mu \mathrm{m} \mathrm{s}^{-1}$ to $1200 \mu \mathrm{m} \mathrm{s}^{-1}$ to select free parameters in our model and test whether our model was capable of reproducing this result. We set $\varepsilon=0$ to eliminate the pulsatile flow effect from this bench mark simulation. In addition, we also investigated clot development under blood flows with different viscosity values $(\mu=0.04,0.02,0.01,0.005$ Poise $)$. We were able

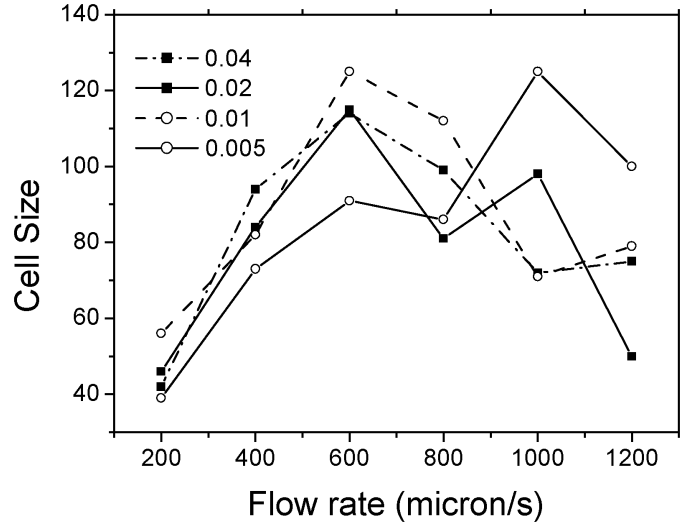

Fig. 4 The effect of blood flow rate on clot growth. The clot size refers to the number of platelets the thrombus is composed of at time $25 \mathrm{~s}$.

to reproduce the trend of the clot growth and show that it was of the same order of magnitude as in the experiment.

Fig. 4 shows distribution of sizes of simulated clots in numbers of coagulated platelets obtained after $25 \mathrm{~s}$, for different blood flow velocities and viscosities. For normal blood viscosity of $\mu=$ 0.04 Poise, the simulation result is very similar to the experimental result described in ref. 9 (Fig. 4b). Simulations demonstrated that growth of the thrombus was strongly affected by two competing factors: the rate at which platelets were supplied by the blood flow to the thrombus, and intensity of shear force which prevented platelets from adhering to the thrombus. When the blood flow rate is low, platelets supply rate plays dominant role. When blood flow rate is high, the shear force increases to such an extent that it prevents platelets from adhering to the thrombus.

Simulations also demonstrated that growth of thrombi was not greatly influenced by the blood viscosity. In a reasonably wide range of values of viscosity $(0.01 \leq \mu \leq 0.04)$, growth of thrombi showed similar characteristics.

\subsection{Clot growth under Newtonian pulsatile flow}

Fig. 5 displays clot size $v s$. various flow rates, viscosities and pulse strengths. Notice that under the normal blood viscosity ( $\mu$ $=0.04$ Poise), clot development has similar characteristics. For the pulse strength varying between 0.0 and 0.6 , the maximum of the clot size occurs at the flow rate between $400 \mu \mathrm{m} \mathrm{s}^{-1}$ and 600 $\mu \mathrm{m} \mathrm{s}^{-1}$. On the other hand, for the extremely low viscosity $(\mu=$ 0.005 Poise), clot development has different characteristics. When there is no pulse, clot size first increases with respect to the increment of the flow rate, then at flow rate of $1000 \mu \mathrm{m} \mathrm{s}^{-1}$ it reaches its maximum, and then starts to decrease. However, clot size keeps increasing as the flow rate increases for the pulse strength of $\varepsilon=0.2,0.4$ and 0.6 respectively.

For the fluid viscosity between 0.04 Poise and 0.005 Poise, the pulsatile flow changes the dynamics of the clot development quantitatively but not qualitatively. This means that for different pulse strength, the maximum of the clot size occurs at different flow rates. Nevertheless, for viscosity $\mu=0.02$ and 0.01 Poise, the size of the resulting thrombus still first increases and then decreases with increase of blood flow velocity. 

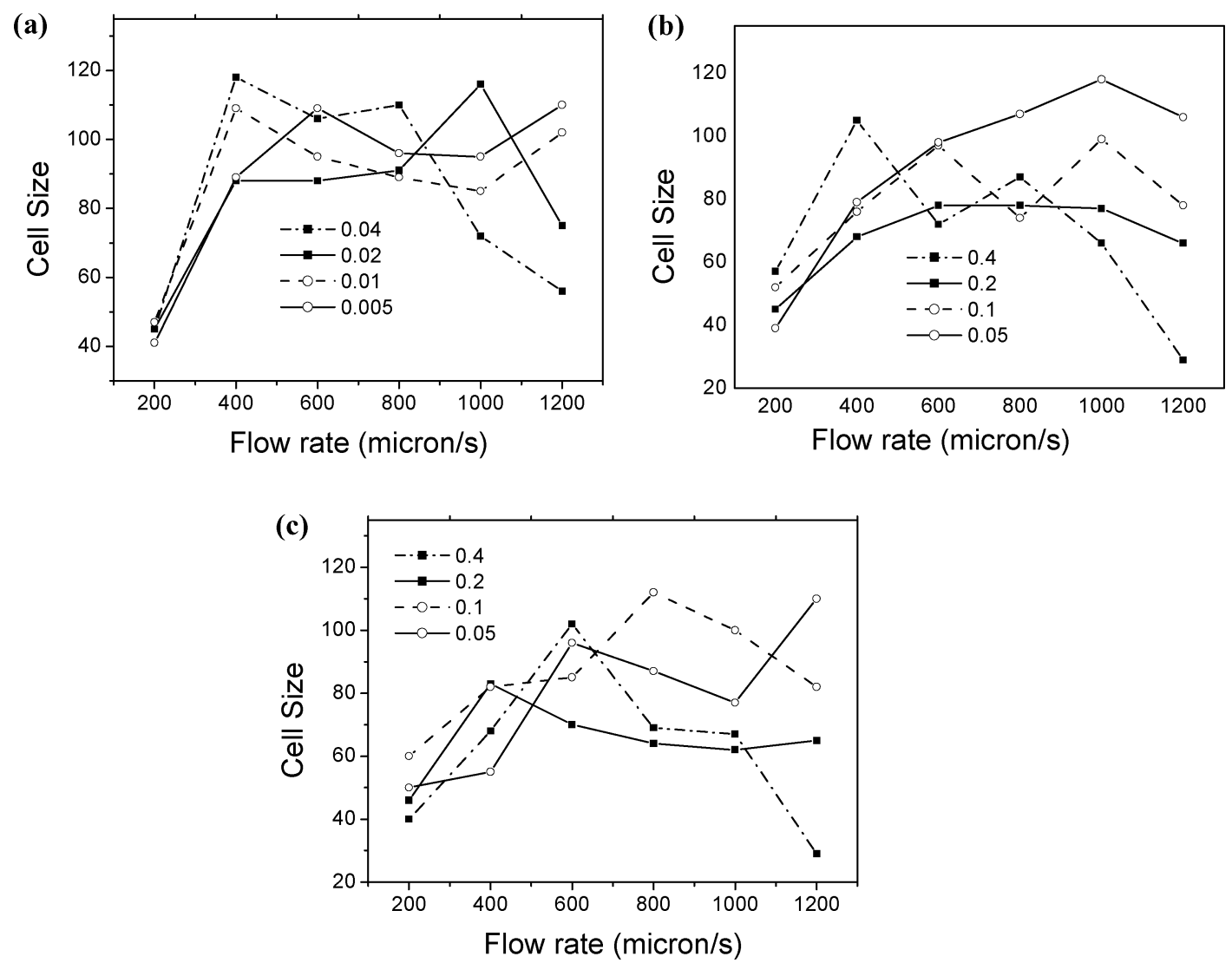

Fig. 5 The effect of pulse parameters and viscosity on clot growth. The pulse strengths are: (a) $\varepsilon=0.2$, (b) $\varepsilon=0.4$, and (c) $\varepsilon=0.6$ respectively. The clot size is given in number of platelets in the thrombus after $25 \mathrm{~s}$.

\subsection{Clot growth under non-Newtonian pulsatile flow}

Fig. 6 displays clot size vs. various flow rates for pulse strengths $(\varepsilon=0.0,0.1$ and 0.2 ). After comparing with Fig. 4 we observe that, in the absence of a pulse, non-Newtonian flow effect is negligible within the range of flow rates we study. Moreover, when pulse strength is small $(\varepsilon=0.1)$, the growth of the clot is not affected by the pulsatile flow. However, increase in the strength of the pulsatile flow changes clot size dramatically. When pulse

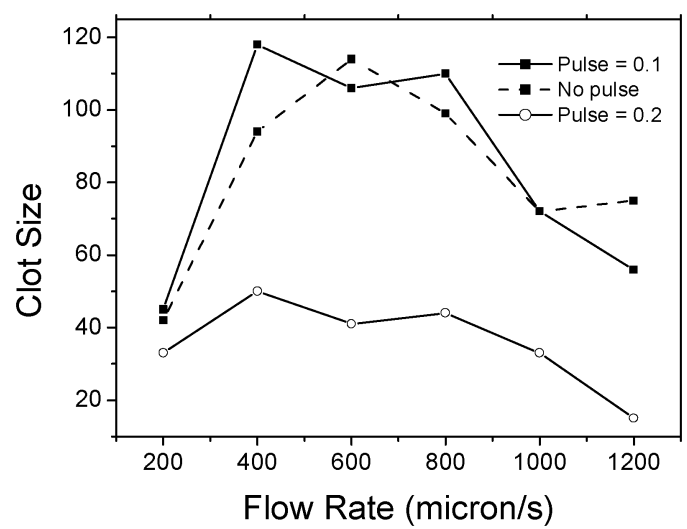

Fig. 6 The effect of non-Newtonian flow on clot growth. The pulse strength is $\varepsilon=0.0,0.1,0.2$ respectively. Clot size refers to the number of platelets the clot is composed of after $25 \mathrm{~s}$. strength is $\varepsilon=0.2$, clot size is significantly smaller. (See Fig. 6 for a comparison.)

\subsection{Distribution of cells in clots formed with different flow rates}

Clots in Fig. 7 and Fig. 8 simulated for the flow rates from $0.1 \mathrm{~cm}$ $\mathrm{s}^{-1}$ to $20.0 \mathrm{~cm} \mathrm{~s}^{-1}$ all have inhomogeneous internal structure. At the same time, the positions of clusters of blood cells are very different. Namely, most blood cells are located in the middle of the clot in Fig. 7a, in the upper part of Fig. 7b and in the lower part of Fig. 7d. This shows that formulation of the internal clot structure is significantly influenced by the blood flow.

To simplify the analysis of the clots' internal structure we divide it into two equal parts (front and back) and compare numbers of blood cells in those parts. For low blood flow rates $\left(0.1 \mathrm{~cm} \mathrm{~s}^{-1}\right.$ and $\left.1 \mathrm{~cm} \mathrm{~s}^{-1}\right)$, the number of blood cells in the front part is higher than in the back part (the ratio is higher than 1.0). For high blood flow rates $\left(>1.0 \mathrm{~cm} \mathrm{~s}^{-1}\right)$ the ratio is close to 1.0 , implying that blood cells are homogeneously distributed in the front and back parts. For a flow rate of $20.0 \mathrm{~cm} \mathrm{~s}^{-1}$, flow vorticity is observed to bring some platelets to the back side of the clot. However, the flow vorticity is weak and flow does not flush larger blood cells to the back side of the clot.

The effect of the low rate on the complexity of the clot structure is observed in the development of thrombi generated following vascular injury in experimental animals. Fig. $7 \mathrm{f}$ is a frame from a video monitoring a single optical plane through 

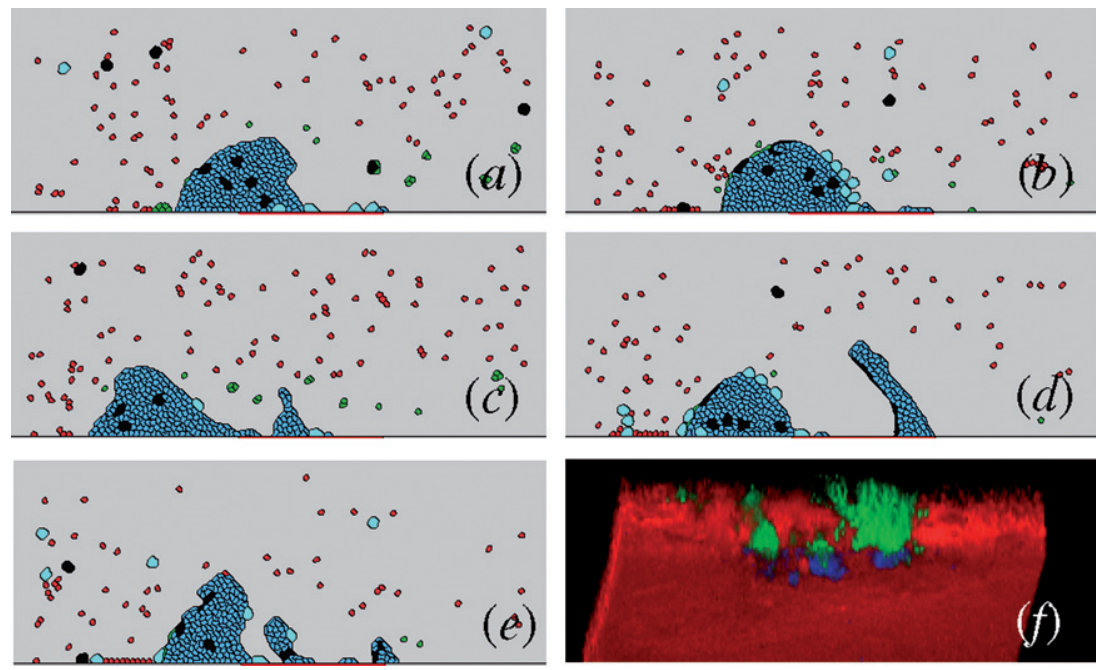

Fig. 7 The effect of flow rate on the blood cell distribution inside the clot. Flow rates $v$ are (a) $v=0.1 \mathrm{~cm} \mathrm{~s}^{-1}$.

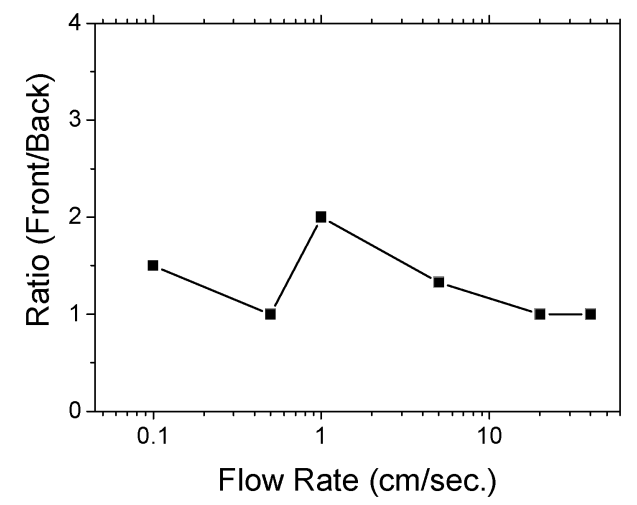

Fig. 8 The effect of flow rate on the blood cell distribution inside the clot. For each simulation in Fig. 7, we divide the clot into two equal front and back parts. The flow is from the left (front part) to the right (back part). The ratio $r$ is defined by $r=N_{\text {front }} / N_{\text {back }}$, where $N_{\text {front }}$ and $N_{\text {back }}$ represent numbers of blood cells in the front and back parts of the clot, respectively.

a clot developing in a mouse mesenteric vessel following a laser induced injury. The frame shows a heterogeneous structure that includes platelets (green) and fibrin (blue) as well as blood cells (black "holes" excluding dextran (red) in blood). The role of perturbations in the flow field leading to the entrapment and incorporation of blood cells and the irregular thrombus structure is more evident in the video recording than in a single frame. The ability of the simulation to predict the effect of flow on clot structural features is the subject of future studies.

\subsection{Analysis of the blood flow near the clot}

Here we apply the methodology described in Section 3.5 to understand the role of the blood flow near the clot. Fig. 9 plots the forward-time FTLE field at 6 instances during the clot formation stage. There is a well-defined repelling LCS that exists throughout the clotting process, which is defined by the curve of high FTLE (shown in red). Proximal to the clot, the LCS remains close to the vessel wall. Nearing the clot, the LCS extends toward the center of the vessel and encloses a region that contains both portions of the blood clot. This LCS appears to terminate on the distal portion of the clot, away from the vessel wall. Distal to the second portion of the clot, there exists a separate LCS, which terminates on the vessel. The integration time used to compute the FTLE fields was $\tau=0.3 \mathrm{~s}$.

In the case where the peak free-stream flow speed is $0.1 \mathrm{~cm} \mathrm{~s}^{-1}$, there is no recirculation present behind the clot throughout the clotting process. The Reynolds number for this flow is $R e=$ 0.001 , based on the vessel diameter and average flow speed. Therefore, the flow is highly viscous and is able to remain attached downstream from the clot.

For the case where the peak free-stream flow speed is $5 \mathrm{~cm} \mathrm{~s}^{-1}$, two clots that are initially separate, but close, form and quickly combine to form a single clot. As these clots develop, an interior region of recirculation exists between them. This region is bound by the repelling LCS shown in Fig. 9 that propagates proximal to the clot region close to the vessel wall and covers the majority of the region where the clot develops. Therefore no transport occurs between this interior recirculation region and the surrounding blood flow, except entrainment of flow near the vessel wall. This thin layer of blood bound by the LCS proximal to the clotting region also constitutes fluid that eventually grows the clot. Distal to the clot, there is a small region of recirculation. This small recirculation region is partially bound by a separate repelling LCS that terminates on the vessel wall downstream from the clot. This LCS is separate from the primary LCS bounding much of the clotting region. The gap between this LCS and the distal side of the clot permits entrainment and detrainment between this small region of recirculation behind the clot and the ambient flow. However, the rate of entrainment and detrainment is small during the clotting process and does not appear to affect the clot formation significantly.

\section{Conclusions}

Since the hemostatic system has evolved to stem the loss of blood following damage to blood vessels, understanding the parameters affecting clot structural integrity has important biomedical 


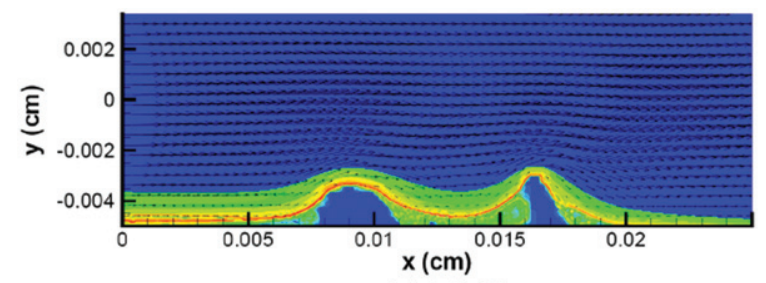

(a) $\mathrm{t}=0.33 \mathrm{~s}$

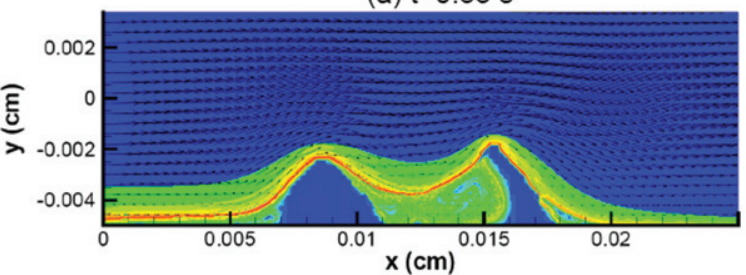

(c) $\mathrm{t}=0.55 \mathrm{~s}$

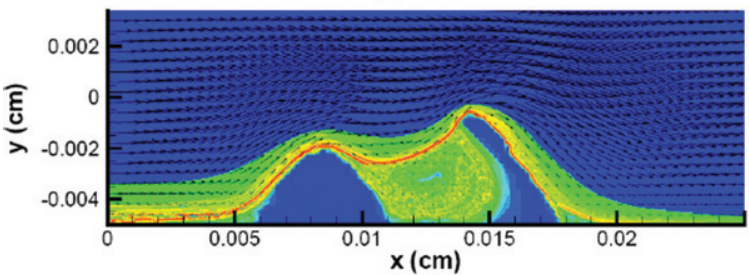

(e) $\mathrm{t}=0.77 \mathrm{~s}$

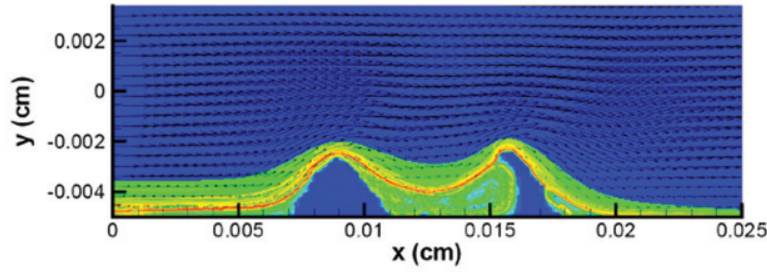

(b) $t=0.44 \mathrm{~s}$

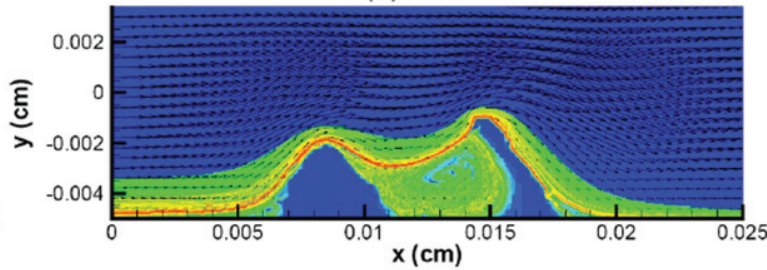

(d) $t=0.66 \mathrm{~s}$

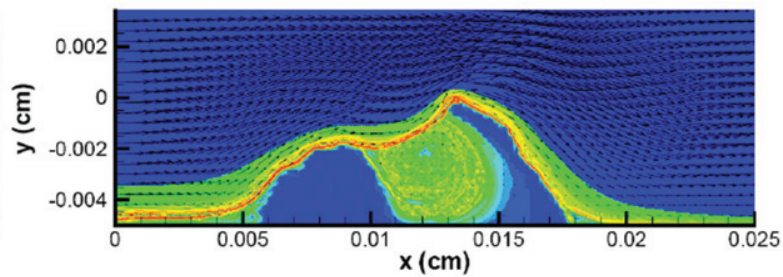

(f) $\mathrm{t}=0.88 \mathrm{~s}$

Fig. 9 Forward-time FTLE field at 6 instances during clot formation. A well-defined LCS is present that defines the boundary between the region of blood propagating downstream and blood that recirculates and clots. The integration time used to produce these plots was $\tau=0.3 \mathrm{~s}$.

implications. Furthermore, intravascular clots formed in response to damage that does not cause vessel rupture presents potentially serious pathological conditions. These intravascular clots (thrombi) can grow and restrict blood flow, causing pathology in the tissues supplied by the vessel. Additionally, unstable thrombi can shed emboli (clot fragments) that can embed in vessels of the lungs or brain potentially causing pulmonary embolisms or ischemic strokes, respectively. These conditions result in a significant number of deaths in the US, and the rate is expected to increase as the population ages.

This paper describes the incorporation of additional hemodynamic complexity into a computational model of thrombus development first introduced in ref. 38. Simulations using this updated model indicate that increasing flow rates lead to greater structural heterogeneity. These irregular clots include domains with distinct compositions and with different mechano-elastic properties. Differential compression by adjacent domains in response to changing forces on the thrombus can lead to fractures at the interface between domains.

We used LCS to determine the region of blood that is transported to the clot. (We only considered the kinematic affect of the blood flow on transport near the clot.) The computation of LCS can also be applied to three-dimensional data, ${ }^{20}$ as has already been done in other cardiovascular applications. ${ }^{34}$ Qualitatively, we expect future computations of LCS using threedimensional blood clot formation data will reveal similar regions of recirculation and transport mechanisms. The typical Reynolds numbers for flow around the clots considered here are sufficiently low that we do not expect any transition to turbulence for threedimensional flow. However, the rough surface of the blood clot often leads to complex secondary instabilities for the flow near the clot. Moreover, previous experience in applying these computation to three-dimensional blood flow data leads us to expect that the three-dimensional simulations may reveal more complex stirring of the blood in the vicinity of the clot due to the increase degree of freedom of transport. This hypothesis will be investigated further in our future work on three-dimensional blood clot formation simulations.

We are currently working on a three dimensional extension of the multiscale approach and incorporating into the model platelet specific dynamics and the biochemical effects influencing platelet transport near the clot boundary. To study these dynamics, a much more highly resolved understanding of the flow must be obtained near the clot boundary, which requires a fully resolved three-dimensional calculation.

\section{Acknowledgements}

This work was partially supported by NSF Grant DMS-0800612 to MA, ZX and NC and by NIH grant HL073750 to EDR.

\section{References}

1 S. Amornsamankul, B. Wiwatanapataphee, Y. H. Wu and Y. Lenbury, Intl. J. of Biomedical Sciences, 2006, 1(1), 42-46.

2 M. Anand, K. Rajagopal and K. R. Rajagopal, Pithily Haemost Thromb, 2005, 34(2-3), 109-120.

3 A. Anderson, M. Chaplain and K. Rejniak, Single Cell-Based Models in Biology and Medicine, Birkhaser, 2007.

4 S. A. Baldwin and D. Basmadjian, Ann Biomed Eng, 1994, 22(4), $357-$ 370.

5 I. A. Barynin, I. A. Starkov and M. A. Khanin, Izv Akad Nauk Ser Biol, 1999, 1, 59-66.

6 E. Beltrami and J. Jesty, Proc Natl Acad Sci U S A, 1995, 92(19), 8744-8748.

7 E. Beltrami and J. Jesty, Math Biosci, 2001, 172(1), 1-13. 
8 R. B. Bird, R. C. Armstrong and O. Hassager, Dynamics of Polymer Liquids, 2nd edn, Wiley, New York, vol. 1, 1987.

9 N. Begent and G. V. Born, Nature, 1970, 227, 926-930.

10 C. H. Brown, L. B. Leverett, C. W. Lewis, C. P. Alfrey and J. D. Hellums, J Lab Clin Med, 1975, 86, 462-471.

11 R. Chaturvedi, C. Huang, B. Kazmierczak, T. Schneider, J. Izaguirre, T. Glimm, B. Hentschel, J. Glazier, S. Newman and M. S. Alber, J. R. Soc. Interface, 2005, 2(3), 237-253.

12 T. M. Cickovski, C. Huang, R. Chaturvedi, T. Glimm, H. Hentschel, M. S. Alber, J. A. Glazier, S. A. Newman and J. A. Izaguirre, IEEE/ ACM Trans. Comput. Biol. Bioinformatics, 2005, 2(4), 273-288.

13 T. M. Cickovski, K. Aras, M. Swat, R. Merks, T. Glimm, H. Hentschel, M. S. Alber, J. A. Glazier, S. A. Newman and J. A. Izaguirre, Computing in Science and Engineering, 2007, 9(4), 50-60.

14 A. L. Fogelson, SIAM JAM, 1992, 52, 1089-1110.

15 S. Goto, Y. Ikeda, E. Saldivar and Z. M. Ruggeri, Journal of Clinical Investigation, 1998, 101(2), 479-486.

16 G. Haller, Physica D, 2001, 149(4), 248-277.

17 Y. Jiang, J. Pjesivac-Grbovic, C. Cantrell and J. P. Freyer, Biophys J, 2005, 89(6), 3884-3894.

18 M. A. Khanin and V. V. Semenov, J. Theor Biol, 1989, 136(2), 127-134

19 M. A. Knewitz and J. C. M. Mombach, Comput Biol Med, 2006, 36(1), 59-69.

20 F. Lekien, S. C. Shadden and J. E. Marsden, Journal of Mathematical Physics, 2007, (6), 065404.

21 E. S. Lobanova and F. I. Ataullakhanov, Phys. Rev. Lett., 2003, 91, 138301.

22 R. Merks, S. V. Brodsky, M. S. Goligorksy, S. A. Newman and J. A. Glazier, Dev Biol, 2006, 289(1), 44-54.

23 S. Newman, S. Christley, T. Glimm, H. Hentschel, B. Kazmierczak, Y. Zhang, J. Zhu and M. S. Alber, Curr. Top. Dev. Biol., 2008, 81, in press.

24 M. Newman and G. Barkema, in Monte Carlo Methods in Statistical Physics, Oxford University Press, Oxford, 1999.
25 M. V. Ovanesov, J. V. Krasotkina, L. I. Ul'yanova, K. V. Abushinova, O. P. Plyushch, S. P. Domogatskii, A. I. Vorob'ev and F. I. Ataullakhanov, Biochim Biophys Acta, 2002, 1572(1), 45-57.

26 R. T. Pierrehumbert, Physics of Fluids A - Fluid Dynamics, 1991, 3(5), 1250-1260, Part 2.

27 A. J. Reininger, H. F. G. Heijnen, H. Schumann, H. M. Specht, W. Schramm and Z. M. Ruggeri, Blood, 2006, 107(9), 3537-3545.

28 P. Riha, F. Liao and J. F. Stoltz, Clin Hemorheol Microcirc, 1997, 17(4), 341-346.

29 Z. M. Ruggeri, J. N. Orje, R. Habermann, A. B. Federici and A. J. Reininger, Blood, 2006, 108(6), 1903-1910.

30 S. C. Shadden, A dynamical systems approach to unsteady systems (PhD thesis), California Institute of Technology, 2006.

31 S. C. Shadden, J. O. Dabiri and J. E. Marsden, Physics of Fluids, 2006, 18(4), 047105

32 S. C. Shadden, K. Katija, M. Rosenfeld, J. E. Marsden and J. Dabiri, Journal of Fluid Mechanics, 2007, 593, 315-331.

33 S. C. Shadden, F. Lekien and J. E. Marsden, Physica D, 2005, 212(34), 271-304.

34 S. C. Shadden and C. A. Taylor, Annals of Biomedical Engineering, 2008, 36(7), 1152-1162.

35 E. N. Sorensen, G. W. Burgreen, W. R. Wagner and J. F. Antaki, Ann Biomed Eng, 1999, 27(4), 436-448.

36 O. Sozinova, Y. Jiang, D. Kaiser and M. S. Alber, Proc Natl Acad Sci $U S A, 2005$, 102(32), 11308-11312.

37 O. Sozinova, Y. Jiang, D. Kaiser and M. S. Alber, Proc Natl Acad Sci $U S A, 2006, \mathbf{1 0 3}(46), 17255-17259$.

38 Z. Xu, N. Chen, M. M. Kamocka, E. D. Rosen and M. S. Alber, J. R. Soc. Interface, 2008, 5, 705-722.

39 V. I. Zarnitsina, A. V. Pokhilko and F. I. Ataullakhanov, Thromb Res, 1996, 84(4), 225-236.

40 V. I. Zarnitsina, A. V. Pokhilko and F. I. Ataullakhanov, Thromb Res, 1996, 84(5), 333-344. 